\section{Mitigating Bias in Big Data for Transportation}

\author{
Greg P. Griffin, Megan Mulhall, Chris \\ Simek, and William W. Riggs
}

This is a pre-print of an article published in Journal of Big Data Analytics in Transportation. The final authenticated version is available online at: https://doi.org/10.1007/s42421-020-00013-0.

Griffin, G. P., M. Mulhall, C. Simek, W.W. Riggs. Mitigating Bias in Big Data for Transportation. Journal of Big Data Analytics in Transportation. doi: 10.1007/s42421-020-00013-0.

\begin{abstract}
Emerging big data resources and practices provide opportunities to improve transportation safety planning and outcomes. However, researchers and practitioners recognise that big data from mobile phones, social media, and on-board vehicle systems include biases in representation and accuracy, related to transportation safety statistics. This study examines both the sources of bias and approaches to mitigate them through a review of published studies and interviews with experts. Coding of qualitative data enabled topical comparisons and reliability metrics. Results identify four categories of bias and mitigation approaches that concern transportation researchers and practitioners: sampling, measurement, demographics, and aggregation. This structure for understanding and working with bias in big data supports research with practical approaches for rapidly evolving transportation data sources.
\end{abstract}

\section{Keywords}

big data, bias, transportation, safety, interview

\section{Introduction}

Obtaining high-quality data for transportation safety planning has been expensive and slow. Recently, new big data sources allow a more detailed analysis of vehicle, transit, bicycle, and pedestrian trips than ever before. However, big data generally represent electronic devices or services rather than individual human behaviour-inherently including a range of biases (Batty, 2018; Shearmur, 2015). Sources of big data sources offer both prospect and problems for transportation planning, regarding how well they reflect the population of transportation system users, or individual markets subject to the digital divide. Rather than merely identify biases in big data, scholars have already suggested that new approaches "must be able to mitigate against these risks" (Harris et al., 2017, p. 609). Research identifies far-reaching bias issues in big data sources, but this study will focus on those with an impact for transportation safety planning. Through a synthetic literature review and interviews with expert practitioners, results from this study suggest implications for transportation safety research and practice to identify and mitigate bias in big data.
This project addresses two critical questions:

1. What are the sources of bias in big data for transportation safety planning?

2. What are approaches to mitigating bias in big data for passenger vehicles, transit, bicycling, and pedestrians?

One practical definition of big data in a planning context is that "it is too large and too complex to be stored, transferred, shared, curated, queried, and analysed by traditional processing applications. There is no specific size assigned to big data, as it is always growing" (Desouza \&
About the authors: Greg P. Griffin (greg.griffin@utsa.edu) is an Assistant Professor at the University of Texas at San Antonio. Megan Mulhall (mulhallm@michigan.org) is a Research Specialist at Michigan Economic Development Corporation. Chris Simek (c- simek@tamu.edu) is an Associate Research Scientist at the Texas A\&M Transportation Institute. William W. Riggs (william.riggs@usfca.edu) is Program Director and Assistant Professor at the University of San Francisco. 
Smith, 2016). Most conceptions of big data include dimensions of the volume of data starting in the terabytes; velocity ranging from very recent to real-time, and variety including multiple data formats that may be highly structured or informal, like social media. Some suggest "the volume of data continues to double every three years as information pours in from digital platforms, wireless sensors, and billions of mobile phones" (Henke et al., 2016). Targeting practical applications for safety, we define big data for transportation as data with applications for the movement of goods or people that requires computing power beyond typical personal applications, through the processes of data collection, warehousing, or analysis.

Big data is changing the way transportation planners work, leading to questions and challenges of justice in how data is used, with recent scholarship suggesting substantial changes are necessary to mitigate potential problems (Schweitzer \& Afzalan, 2017). For example, planners use fitness app data to understand cycling routes, but studies have shown the big data sources provide only a segment of the population, varying significantly from survey data using traditional sampling methods (Bergman \& Oksanen, 2016b). Similarly, comparisons of several big data sources on the same routes show significant differences, representing a fraction of total travel (Griffin \& Jiao, 2015a). Big data often results as a 'residue' of electronic activity, may have substantial privacy issues, and rarely aligns to specific urban problems without substantial processing (Badu-Marfo, Farooq, \& Patterson, 2019; Batty, 2016).

Despite these challenges, big data "can reveal new dynamics, can allow for the study of certain processes in real time and can highlight relationships and correlations that may pass unnoticed using classical methods and data" (Shearmur, 2015). Big data can lead to insights at spatial and temporal scales not previously possible (Hong, Kim, \& Widener, 2019; Zhou, Chen, Yeh, \& Yue, 2019). Through new insights on the use of big data in transportation and smart cities, this study supports both research and practice of transportation planning. The next section describes recent developments in big data for transportation and documented research needs. Following the background, we describe this study's method, results, and conclusions.

\section{Literature Review}

Research on big data in transportation expanded since 2010, and continues to increase across sub-fields in safety and analytics. We searched research using Scopus, TRID, and Science Direct to identify research related to sources and mitigation of bias. Search terms in these databases included "big data," "transportation," and "safety," resulting in 135 publications addressing this study's research questions since the year 2010. We then supplemented this search with Google Scholar to find seminal and recent work citing key papers. Among these, the 75 studies addressed this study's research questions considering six issues: methodology, data type, bias sources, mitigation techniques, topics (transportation planning, planning in general, safety, and smart cities), and transportation mode (transit, surface freight, automobile, bicycle, and pedestrian).

Research suggests that big data, collected by sensors that are part of transportation infrastructure, vehicles, cargo, or people, offer promise to help answer questions about transportation safety that could save lives. Transportation modes can be inferred from mobile phone and other data (Efthymiou, Barmpounakis, Efthymiou, \& Vlahogianni, 2019; Stenneth, Wolfson, Yu, \& Xu, 2011). Connected vehicle data may greatly reduce the time necessary for identifying high-risk locations, as compared with historical crash data (Xie, Yang, Ozbay, \& Yang, 2019). Smart cards used for transit systems offer insights for passenger market segmentation (Kieu, Bhaskar, \& Chung, 2015). Some sensors, such as 24-hour cameras, provide relatively unbiased data consistently if the stream can be parsed into useful information (Hipp et al., 2017). However, most big data represent items rather than people, such as smartphone or vehicle locations and trajectories. "However big the data, Big Data are not about society, but about users and markets" (Shearmur, 2015). The opportunity for transportation safety, however, is that big data allows the analysis of changes nearly instantly, and the data may reveal fine-grained spatial and temporal relationships that are invisible with traditional methods.

Big data and traditional sources for transportation safety may include different forms 
of bias, which might distort the analysis (GarcíaAlbertos, Picornell, Salas-Olmedo, \& Gutiérrez, 2018). Mobile phone location and activities aggregated for transportation use typically only include data when network connections are present and specific activities are recorded, described as a "potential activity bias" (Diao, Zhu, Ferreira, \& Ratti, 2016, p. 938). Additionally, big data from mobile phone locations can obscure trips in which the origin and destination are within the same cellular tower range (Zhou, Yeh, Li, \& Yue, 2018). However, big data sources may fill gaps in needed information, as well. For instance, transportation agencies collect bicycling and pedestrian volume data sparingly, if at all, creating a challenge for understanding relative safety risks (Piwek, Joinson, \& Morvan, 2015). Big data also supports new approaches for managing freight modes, including maritime shipping (Peng et al., 2018). Big datasets such as online street-level imagery can potentially be used for collecting pedestrian volumes, but do not currently include time-of-day information to support scaling over known time periods (Yin, Cheng, Shao, Wang, \& Wu, 2017). In travel demand modelling, practitioners leverage the large sampling sizes of origindestination big data, combined with locational demographics from census data - combining the strengths of each data type (Erhardt \& Dennett, 2017). Big data from probe vehicles offers realtime speed data to manage traffic congestion and incident management (Brennan, Gurriell, Bechtel, \& Venigalla, 2019). Research exploring how to mitigate biases, such as those related to various problems with spatial aggregation and self-dependency are needed (Abdel-Aty, Lee, Siddiqui, \& Choi, 2013; J. Zhao, Wang, Xing, Luan, \& Jiang, 2018).

Emerging transportation technologies, such as mobility-as-a-service (MAAS), and connected and automated vehicles, might leverage big data to improve safety and other outcomes (Krishnamurthy, Smith, \& Desouza, 2017; Legacy, Ashmore, Scheurer, Stone, \& Curtis, 2019; Xie et al., 2019; Zmud et al., 2018). Transportation network companies also use big data to predict demand and allocate drivers and vehicles efficiently. Increases in big data-driven mobility services could "improve road safety by creating a more viable option that keeps people from getting behind the wheel when they are ... impair[ed]" (Henke et al., 2016). Researchers are applying data mining techniques to understand safety problems across modes, such as tram faults (Gürbüz \& Turna, 2018). However, some sources and applications of big data may have ethical issues. For instance, algorithmic selection of individuals for passenger airline no-fly lists might inadvertently restrict personal rights (Shearmur, 2015). "Hidden biases in both the collection and analysis stages present considerable risks and are as important to the big-data equation as the numbers themselves" (Crawford, 2013).

Review of big data literature and 75 studies specific to big data in transportation, shows early emphasis on studies of transit. Ten of the transit studies leveraged social media data, employing analysis of text to study perceptions, and geotagging to ascertain location and time. The prominence of social media in these studies may happen to parallel a broader inquiry of the rise of social media in contemporary life, in addition to the use of social media as transit riders. We also found safety analysis with other travel modes: surface freight, automobile use including taxis, bicycling, and walking. Additionally, we found related studies in transportation planning, planning in general, and smart cities addressing issues of bias in big data that applied to this study. We interweave findings and significance from our review of the literature with results from interviews with experts.

\section{Interview Methods}

Following our review of previous research, we interviewed experts to capture ideas on our research questions, beyond previously published work. We constructed a guide for semi-structured interviews to focus on insights from expert practitioners of big data in transportation. The semi-structured interview approach allows the interviewer to focus the discussion on topics of interest while encouraging the interviewee to emphasise particular interests and expertise (Adams, 2015). We included "probes" as bulleted items on the guide, related to our research questions. Some probes are expected to fit some interviewees more than others, providing additional flexibility to the discussion.

\section{Interview Candidates}

Interview candidates were identified using the search term "big data" in conference agendas 
for conferences related to transportation and planning: Transportation Research Board (TRB) Annual Meetings in 2017 and 2018, and the American Planning Association (APA) National Planning Conference held in 2017. From these results, interview candidates were chosen using the following criteria: a) presenting research or participating in roundtable discussion of big data, b) holding a position within their organisation suggesting substantial knowledge big data applications, and c) current contact information is readily accessible through conference agenda or online. We sought interviewees with knowledge of and experience applying the techniques necessary to mitigate bias encountered in big datasets, allowing us to capture candidates such as a travel demand modeller at a state department of transportation, as well as individuals that sell transportation datasets for planning purposes.

We requested interviews via email with thirty-nine experts in the use or analysis of big data for transportation. Ten respondents completed interviews, resulting in a response rate of $26 \%$. Interviewees worked in one of four categories: academia $(\mathrm{N}=4)$, private sector $(\mathrm{N}=3)$, state departments of transportation $(\mathrm{N}=2)$, and city transportation department $(\mathrm{N}=1)$. Interviewees spanned much of North America, including three from California, two in Georgia, and one each from Texas, Wisconsin, Virginia, the District of Columbia, and Ontario (Canada). To encourage candid responses, we offered anonymity.

\section{Interview Protocols}

Interviews were conducted online, using text only (with one exception of a participant who preferred to use full audio and video). Advantages to using online and using text-only interview methods include not requiring transcription, there are no audio or video transmission problems, and it is convenient because it provides little interruption for the interviewee (O'Connor, Madge, Shaw, \& Wellens, 2008). However, online text-based interviewing lacks information from non-verbal communication and nuances in speech, in addition to slowing the speed of communication via typing, as compared with real-time speech. The full interview dataset is available for additional analysis (ANONIMIZED).

\section{Coding and Analysis}

Despite the relatively small dataset, key issue coding allows a review of content in a consistent method focusing on central themes (Ose, 2016). Coding of qualitative data, such as interviews, requires "observers or readers to categorise, scale, or measure each of a given set of predefined units of analysis, in effect characterising them by one value from each variable of analytical interest. However, there are many occasions in which texts... have multiple interpretations" (Krippendorff \& Craggs, 2016). We use applied thematic analysis with predefined codes, and acknowledge that this method may omit some meaning intended by a respondent, but accept this limitation for pragmatic, rather than interpretivist application (Guest, MacQueen, \& Namey, 2014). This study employs a coding analysis method that enables the evaluation of the reliability of multiple codes to each interview question response by two analysts, resulting in an overall reliability coefficient (Krippendorff \& Craggs, 2016).

Interview coding involved three steps; developing a codebook to guide analysis of interviews, coding of the interview data itself, and preparation for reliability analysis. The codebook focuses only on fundamental concepts related to this study's research questions, described in qualitative analysis research as structural code analysis (Guest et al., 2014). Interview content was then formatted into de-identified spreadsheets, with responses to each question on a new row for review by the coders. Two researchers who were not involved with the interviews then independently coded the transcripts, reviewing each response for the occurrence of any of the seven codes. The coding analysis mirrors a double-blind review since neither the coders nor interviewees knew each other. Briefly, the seven codes were: "source" relating to something that causes some biased understanding of transportation; "mitigate" included a way a respondent mentioned dealing with issues of bias; four passenger travel modes of "vehicles", "transit", "bicycling" and "walking"; and a code of "interpreting" that signified an interviewee's description of how they understand big data as a concept or technology. Analysts reviewed these codes across each question for all interviewees. Reliability was assessed using open-source Multiple-Valued 
Nominal Alpha software, available from https://github.com/rcraggs/mvna/releases.

\section{RESULTS}

\section{Interviews}

Experts responded to our interview prompts with insights ranging between two and more than 200-word responses to individual questions. Interviewees reported starting work with big data as early as 2006, and as recently as 2014 . However, a lead city traffic manager noted that "it depends on how you define 'big data'. I've dealt with large-ish data in transportation since 2002, but considered it 'big' starting around 2008". A division director for a state department of transportation described more specifics of what counts as 'big' with some of the new problems associated with having more data.

Our [brand name] system collects speeds, volumes, and occupancy data lane-by-lane every 20 seconds. We have hundreds of those installations around metro [area]. That, to me, is big data. Lots of it, difficult to analyze and draw trends/conclusions from.

Several other interviewees noted challenges of working with big data in transportation, but these issues also create new business markets for consulting. A CEO for a data provider described how they turn the problems of big data into opportunity. "My company gets big data from millions of smartphones and connected vehicles. Then we turn the data into patterns that describe transportation patterns. We build and sell software that allows clients to ask the data questions and get back accurate and privacyappropriate answers."

Our interview coding system allowed an analysis of individual and combinations of codes in a single response. Co-occurrence analysis of topics resulted in 77 combinations of codes found in each response. Overall, the coders agreed on $76 \%$ of individual responses, with a multi-value nominal alpha coefficient $(\mathrm{mvn} \alpha=0.544)$, meaning that coders interpreted individual interview responses the same more than half of the time, after controlling for the probability of agreement due to chance.

Source (of bias) was the most common code in the dataset, identified twenty-three times by the coders. For instance, a respondent employed as a researcher described, "big data from mobile devices probably under-represents older segments of the population and maybe lower income populations. However, it also does a better job of representing under-represented road users like bicyclists and pedestrians". Other respondents also indicated that the presence of bias in big data did not preclude its usefulness, but suggested the importance of recognising and addressing the issue.

Travel mode was another common topic mentioned by interviewees, who mentioned vehicles nine times, bicycling eight times, and transit three times. Interviewees did not explicitly discuss pedestrian travel in the context of big data.

Mitigation (of bias) was coded in nine instances. One interviewee who worked as researcher described a range of ways that big data users can mitigate bias.

Combine big data with 'traditional data' like surveys. Interpolate big data or use it to create synthetic populations. Wait for larger sample sizes. Skilled experts can work with raw data using data mining and machine learning techniques. Less-skilled users can acquire data from intermediaries that make sense of it for them. Even then, they probably need basic data management skills and often GIS capability.

This response suggests that new methods for mitigating bias are still needed. Recent research shows that data fusion can leverage advantages of multiple data sources (Diao et al., 2016; Wagh, Li, Sudhaakar, Addepalli, \& Qiao, 2013), but few big data providers or practitioners currently use them-skill levels limit mitigation of bias (M. Zhao, Venkatanarayana, \& Fontaine, 2017).

Interpretation (of how big data is understood as a concept or technology) was coded eleven times. One researcher described big data as potentially problematic regarding high volumes that may contribute little to understanding, noting, "our cities are getting data-obese. We need to learn how to cure them. I think data quality is an important factor to consider. Data bias is a concerning sign of 
sickness".

\section{Bias in Mobile Phone Data}

Mobile phone data was also frequently mentioned by interviewees and is one of the most widely used big datasets in transportation planning (C. Chen, Ma, Susilo, Liu, \& Wang, 2016). One interviewee described big data as mainly "being generated through cell phones or non-engagement [passive] methods." Since these data are generated passively, recorded each time a mobile phone connects with a tower in the network, integrating this information into the transportation planning process requires significant inference. Because mobile phone data, like call data records, or sightings data, were not explicitly collected for transportation planning, the data often do not answer typical transportation planning questions (Toole et al., 2015). As such, bias can enter the transportation planning process when planners make inferences from cell phonebased big datasets (C. Chen et al., 2016).

Different sources of mobile phone data also vary in types of bias for transportation analysis. Despite the fact that mobile phone data are among the most applied and researched types of big data in transportation planning, some researchers have expressed uncertainty regarding how the data was collected, and how accurately it represents transportation actions (Toole et al., 2015). When a caller is in an area of overlapping mobile base stations, the location identification may not accurately measure location. Additionally, individuals are not identifiable in many areas due to privacy restrictions. Understanding the different "levels of geographic specificity" between common types of mobile phone records is a crucial prerequisite for integrating such big data into transportation planning processes (C. Chen et al., 2016; Taylor, 2016).

Beyond the understanding of how the big data was collected and if they can find ways to contextualise the data in their study communities, researchers and practitioners can address sampling-related biases that make mobile phone data unrepresentative. First, mobile phone ownership rates fundamentally impact who is covered in a mobile phone-based sampling frame. (C. Chen et al., 2016; Taylor, 2016; Toole et al., 2015). Since mobile phone use is not ubiquitous across any population, in addition to varying rates of use by different phone companies, the sampling frame is subject to coverage bias (Bonnel, Bayart, \& Smith, 2015; C. Chen et al., 2016; Taylor, 2016; Toole et al., 2015). Furthermore, if those absent from the data sample differ systematically from those that are present in the sample (mobile phone users) on critical demographics or other inputs, the sample is subject to non-response bias (Bonnel et al., 2015; C. Chen et al., 2016; Taylor, 2016; Toole et al., 2015).

Finally, measurement bias occurs because mobile phone records in the dataset may not accurately describe the indicators of interest to the researcher (Bonnel et al., 2015). For example, big data users cannot assume that one SIM card record in their dataset represents just one targeted user (Taylor, 2016). Multiple users may share one cell phone or SIM card, or one user may have multiple mobile phones or SIM cards (C. Chen et al., 2016; Taylor, 2016). Further, the proximity of mobile phones in the study area may make it difficult for transportation planning researchers to segment individual mobile phone users for inferences such as travel trajectory (C. Chen et al., 2016).

\section{Bias in Social Media Data}

Existing published research covers bias issues with the use of social media for transporation planning, but interviewees did not mention this explicitly. Tass and Hong identified three kinds of sampling bias from the use of geotagged social media data to understand urban dynamics (Tasse \& Hong, 2017). They showed how the use of Twitter and Foursquare represent participants' voluntary actions, rather than strict records of urban movement. For example, "the Museum of Modern Art in New York has more check-ins than Atlanta's airport, even though the airport had almost three times as many visitors in the period that was studied" (Tasse \& Hong, 2017). Second, social media users that provide location data do so to show where they want to be seen, rather than where they travel. Researchers describe this problem as social desirability bias (Beecham \& Wood, 2013; Johnson \& Smith, 2017), in which people provide information to promote personal accomplishments, rather than accurately depict their activity. Third, urbanites in general, and "young, male, technology-savvy people" use social media at higher rates than other 
groups, creating a sampling bias for analysts wishing to understand transportation system users in general (Murphy et al., 2014). Additionally, many posts are made by organisations rather than individuals, and communication through social media may be dominated by frequent posts of some users (Evans-Cowley \& Griffin, 2012; Schweitzer, 2014). Nonetheless, researchers are developing approaches to use social media for a wide range of transportation applications, including methods to adjust for the varying sample of social media users (Zhang \& He, 2019).

\section{Bias in Travel Observation Data}

Previous research revealed examples of big data research dealing with bias in each travel mode. Automobile data collection includes sensors that are part of the vehicle, such as GPS, toll tags including RFID tags, and license plates readable by cameras; and sensors carried by the driver, including smartphones. Interviews suggest many challenges for working with big data regarding vehicle use. A private-sector interviewee described their work as including "a lot of multi-agency systems and traveler information systems, and use data from a variety of sources to integrate them together for better information." As previously suggested, a sampling (or demographic) bias occurs when the people buying the products tracked, like a car or phone, does not represent the total population. Travel surveys using GPS may be more accurate than traditional travel diaries regarding time and routing of trips, but they can introduce problems with correct identification of travel mode and trip purpose (Vij \& Shankari, 2015) — key inputs for travel modelling. However, big data could be particularly useful for tracking complex travel behaviours such as ridesplitting (X. Chen, Zahiri, \& Zhang, 2017). A consultant noted that "local planners have come to us once we have started data warehouses to get data for their needs," suggesting this area as an emerging field that depends making big data resources more refined and accessible.

Bicycling data can come from sensors through a dedicated bicycle computer (combined speedometer, GPS map, and often heart rate or power calculations), in addition to mobile phone apps. Several companies offer sports-oriented platforms that can record the same travel observation data on either a dedicated bicycle computer or a smartphone app (Garmin, 2018; Kitchel \& Riordan, 2014). These datasets provide an opportunity to address shortcomings in transportation data collected by agencies, but the use of the equipment and apps (often expensive) create a significant sampling (or demographic) bias (Bergman \& Oksanen, 2016a). Review of the app users' demographics shows primary representation of male, young, and middle-aged segments of the population (Boss, Nelson, Winters, \& Ferster, 2018; Griffin \& Jiao, 2015a, 2015b). Additionally, bicyclists may choose not to record all of their trips with many of these apps that also provide online social sharing (Smith, 2017) - again incurring a social desirability bias, where participants may only log bicycle trips that could skew faster or longer than the average ride, to record and share as an accomplishment.

Pedestrian travel observation through big data is in its infancy, other than simple counts using automatic detectors (Griffin, Nordback, Götschi, Stolz, \& Kothuri, 2014). One researcher interview confirmed the problem with big data for pedestrian travel "is that there is some imbalance from those represented in big data versus all pedestrians...this could bias against older people who do not use a cell phone." Running tracked via mobile apps are subject to the same bias problems as bicycling data (Bergman \& Oksanen, 2016a), but could be even more exacerbated based on use-walking trips may be too numerous to track using an app that requires activation for each trip. However, approaches to automatically track pedestrian trips using accelerometers or other sensors may support a broad representation of a pedestrian community.

Surface freight is a particularly challenging mode to track, perhaps exacerbated by the proprietary nature of competitive businesses. To protect business interests, big data providers aggregate individual trips and shipments to protect freight carriers' competitive interests. Aggregation of trip data may obscure trip information present in individual data records, resulting in an aggregation bias inherent in analysis products (Mehmood, Meriton, Graham, Hennelly, \& Kumar, 2017).

Transit data sources range widely from automatic counter systems and smart cards that record close to all users of the system-and therefore have little inherent bias-to social media data that each study considers 
unrepresentative of the user population. One analysis of transit reviews found that the demographics of social contributors did not match the target of transit users - they skewed young and affluent (Mondschein, 2015). Additionally, online reviews of transit agencies did not always focus on how transit systems could improve, limiting the utility of this information for system planning and operation.

Despite these biases, interviewees generally reported they support improvements to transportation planning. When asked how big data can help, one consultant stressed speed and cost savings.

Instead of spending bulk of funds on finding data, planners and researchers can now use most of their funds to solve problems using existing data and data fusion platforms. Also, web based data visualization and analytics made planning efforts significantly more efficient and accessible to a larger audience.

Transportation practitioners and researchers were more cautious of the benefits. A transportation professor couched the status of big data as just beginning to reach its stride. "We have seen some benefits, but it is hard to make a conclusion now. I also talked to some other agencies. We are all at the early stages of using Big Data applications. We are still learning about the benefits and risks". Additional studies are needed to evaluate the return on investment of big data for transportation (M. Zhao et al., 2017).

\section{DISCUSSION}

\section{Mitigating Bias in Passenger Vehicle, Bicycling, and Walking Data}

Five different studies described approaches to mitigate bias in big data for motorised vehicles, but the methods may be applicable to data from any mode about transportation safety. First, filtering unreasonable data points could include speed-based or pattern-based filtering methods to flag likely incorrect or inapplicable data for a given circumstance (Bao, Liu, Yu, \& Xu, 2017; C. Chen et al., 2016). One interviewee noted the importance to treat vehicular customers as equals, describing their operational intent as: ...all vehicles are equal and so we work to manage traffic as equally as possible... whether the [data-providing] vehicle is a traditional personally owned vehicle, an Uber in route to a pickup, or an Uber with customer.

Addressing transportation system users as key customers, rather than the vehicles, suggests a need to evaluate how big data can misrepresent travel. Methods to standardize big data using a reference dataset such as census or travel diaries can help control for sampling bias, improving the combined data service's representation of the population (Toole et al., 2015). However, the lack of personal identification and demographics in most big data sources may require integration with census data specific to location, but the uncertain geographic context may introduce further errors (Erhardt \& Dennett, 2017; Kwan, 2012). Another approach to improve representation includes weighting the sample of data against census totals or collecting more data from under-represented users (Mondschein, 2015). Inclusion of high-quality metadata that describes the development and refining of any big data resource helps users address problems with bias (Mcardle \& Kitchin, 2016). Finally, provide ways for data users or consumers to share data cleaning and analysis methods, such as in an online forum (Mcardle \& Kitchin, 2016). Open access to methods for mitigating bias could be particularly helpful for new markets and applications of big data, where the users represent the subject matter experts for how the data should be interpreted and deployed in practice.

These techniques apply to the mitigation of bias in big data, including nonmotorized modes, but the sampling bias in motorised transportation data may not fit nonmotorized modes in the same way. An interviewee who works as a researcher confirmed the presence of bias but saw the utility in big data resources for bicycling and walking. "Big data from mobile devices probably under-represents older segments of the population and maybe lower income populations. But it also does a better job of representing under-represented road users like bicyclists and pedestrians." A consultant who works mostly with data on 
motorised modes countered this notion. "We don't have transit usage, or bicycle usage - so there are parts of the population that don't use cars that are probably underrepresented in their needs." This suggests that any single source of big data might not characterise all travel modes well, but careful use of the data, even if it includes biases, might improve the representation of some transportation system users.

\section{Mitigating Bias in Freight Data}

Surface freight can be challenging to understand, limiting opportunities to mitigate biases in big data. One researcher positioned a significant advantage of big data as supporting more specifics about transportation system movements - key for analysing freight.

In practice and research, big data definitely helps us do research at a much more micro-level than before. Before, the unit of analysis at the census block group was restrictive-big data helps us take a much closer look...Additionally, temporal analysis is more possible, including both longitudinal, and time-of-day analysis; so there are new opportunities here as well.

However, privacy restrictions for big data use also apply to valuable micro-level freight data, which often get aggregated spatially or temporally to protect sensitive information. Markovian approaches can correct for aggregation bias (Mehmood et al., 2017), which may be considered along with other techniques that incorporate a random selection of individual data to detect and adjust fit to the aggregated data.

\section{Mitigating Bias in Transit Data}

Operational characteristics of transit support multiple methods to mitigate bias in big data. Detailed travel monitoring can occur at the point of fare purchases, vehicle boarding, and vehicle alighting. Also, government requirements, such as by the US Federal Transit Agency, requires statistical reporting that enables evaluation of other data sources. For instance, transit fare card data can be validated against traditional travel survey methods like on-board counts and surveys, in addition to understanding through interviews
(Gschwender, Munizaga, \& Simonetti, 2016). Combining qualitative data, such as interviews, with big quantitative data shows a mixedmethods approach to validating big data (Teddlie $\&$ Tashakkori, 2010). Mixed-methods approaches can help answer questions relating to how and why big data should be viewed sceptically or mitigated in specific contexts.

\section{Summary of Bias Mitigation Techniques for Big Data}

Review of the sources and types of bias in big data for transportation suggests issues with using big data without addressing some of its inherent problems. As one researcher we interviewed said, "Our cities are getting dataobese. We need to learn how to cure them."

Broadly, we find four different approaches to mitigate groups of biases that align with four classes of bias seen in big data for transportation, shown in Table 1. Various types of data fusion, including both traditional approaches such as weighting groups by Census statistics, or combinations of new datasets, may be appropriate for biases of population representation. However, when the measurement technique itself does not represent the phenomena - such as assuming only one person uses one mobile phone-filtering data might be required. This type of problem may go unknown or unmitigated without evaluation specific to measurement problems. Traditional counts, surveys, and mixed-method analysis with qualitative data can be helpful with social desirability bias, often found with social media, or demographic biases that could also be related to coverage, sampling, and non-response. Finally, techniques to support privacy by aggregating data often imposes new biases, depending on groupings of time and space. Depending on the data requirements, these might be mitigated with modeling techniques, but these also likely impose errors as well. 
Table 2. Summary of Bias Sources and Mitigation Techniques for Big Data in Transportation
Types of Bias
Mitigation

\begin{tabular}{|c|c|}
\hline $\begin{array}{l}\text { Coverage, Sampling, or Non- } \\
\text { response Bias }\end{array}$ & $\begin{array}{l}\text { Data Fusion (add multiple providers, or weighting with } \\
\text { Census) }\end{array}$ \\
\hline Measurement Bias & Filtering unreasonable data points \\
\hline $\begin{array}{l}\text { Social Desirability or Demographic } \\
\text { Bias }\end{array}$ & $\begin{array}{l}\text { Weighting with traditional counts or surveys, Validation } \\
\text { with mixed methods }\end{array}$ \\
\hline Aggregation Bias & $\begin{array}{l}\text { Spatial or temporal modeling, including Markovian } \\
\text { techniques }\end{array}$ \\
\hline
\end{tabular}

\section{Limitations and Future Research}

Study limitation constrained the total number of interviewees to ten working in transportation fields, and many of our findings may not be generalizable to other disciplines, such as economic studies using market data. Big data research is also changing rapidly, and additional studies with more global representation will likely expand knowledge of bias mitigation techniques.

Big data sources are relatively new, limiting their applications for longitudinal studies (Batty, 2018). However, this suggests new opportunities for future work. Researchers should consider how expansion of mobile phone markets changes data characteristics over time and space for different applications, whether in transportation safety or urban retail, for instance. Additional insights are needed to determine changes in use of big data across different urban science industries, and to assess the impact of these approaches on lived experiences and urban equity.

\section{CONCLUSION \& IMPLICATIONS}

Our analysis finds both challenges to and opportunities for using big data for transportation safety. Through synthesis of literature and interviews, this study sought to provide guidance on addressing bias issues in transportation, focusing first on safety issues. Validation through recent analytical improvements in qualitative coding supported evaluation of critical issues in bias and how to mitigate them in practice and research. Through coding of interview data by independent researchers, we confirmed the reliability of our constructs through agreement on $76 \%$ of individual coded interview responses.
Findings from interviews with experts and recent research suggested four key insights for improving applications of big data for transportation. First, transportation experts and public interests should be central to determine goals and metrics to evaluate transportation safety. Research and expert interviews suggest decisions about which transportation metrics to prioritise should be driven by logical and ethical interest in the public good, rather than convenient applications of new datasets and dashboards. Second, methodological innovations are needed to relate big data to the total population needed for transportation safety. Traditional census data, surveys, and traffic counts are vital to evaluating and factoring who, what, where, and how many crashes occur through big data sources. Third, big data should be used to address key transportation challenges not solvable using traditional approaches. Transportation agencies may lack high-quality information to support data-driven safety planning for bicycling, pedestrian, and emerging transportation modes. Finally, researchers and practitioners should partner on studies to apply emerging knowledge to future challenges. For instance, the transportation safety implications of automated vehicles could be enormous (Buehler, 2018; Crayton \& Meier, 2017; Fagnant \& Kockelman, 2014; Guerra \& Morris, 2018). However, many studies to date use simulated data and conjecture to forecast potential safety impacts. Survey data provides a middle way through, connecting people's experiences to their imaginations for how they will travel in the future-existing ridehailing customers represent a valuable pool of survey participants, since future AV services may follow some aspects of current ridehailing services 
(Sener, Zmud, \& Simek, 2018). Emerging sources big data, such as from ridehailing, could be applied as empirical proxies for technologies that are not currently measurable on a regional scale-such as autonomous vehicles.

This study showed that insights from expert practitioners and researchers may be complementary. Researchers should consider the ethical implications of their big data research on transportation safety, and listen to practitioners for issues that should drive safety outcomes. Practitioners can partner with to researchers to help evaluate new big data resources, recognizing the need to address sources of bias identified in this study. New sources of big data for transportation can potentially save lives with careful application, but their advancements should be critically reviewed before implementation.

\section{References}

Abdel-Aty, M., Lee, J., Siddiqui, C., \& Choi, K. (2013). Geographical unit based analysis in the context of transportation safety planning. Transportation Research Part A: Policy and Practice, 49, 62-75. doi: 10.1016/j.tra.2013.01.030

Adams, W. C. (2015). Conducting SemiStructured Interviews. In Handbook of Practical Program Evaluation (pp. 492505). doi: 10.1002/9781119171386.ch19

Badu-Marfo, G., Farooq, B., \& Patterson, Z. (2019). A Perspective on the Challenges and Opportunities for Privacy-Aware Big Transportation Data. Journal of Big Data Analytics in Transportation, 1(1), 1-23. doi: 10.1007/s42421-019-00001-z

Bao, J., Liu, P., Yu, H., \& Xu, C. (2017). Incorporating twitter-based human activity information in spatial analysis of crashes in urban areas. Accident Analysis and Prevention, 106, 358-369. doi: 10.1016/j.aap.2017.06.012

Batty, M. (2016). Big Data and the City. Built Environment, 42(3), 321-337. doi: 10.2148/benv.42.3.321
Batty, M. (2018). Inventing Future Cities. Cambridge, MA: MIT Press.

Beecham, R., \& Wood, J. (2013). Exploring gendered cycling behaviours within a largescale behavioural data-set. Transportation Planning and Technology, 37(1), 83-97. doi: $10.1080 / 03081060.2013 .844903$

Bergman, C., \& Oksanen, J. (2016a). Conflation of OpenStreetMap and Mobile Sports Tracking Data for Automatic Bicycle Routing. Transactions in GIS, 20(6), 848868. doi: $10.1111 /$ tgis. 12192

Bergman, C., \& Oksanen, J. (2016b). Estimating the Biasing Effect of Behavioural Patterns on Mobile Fitness App Data by DensityBased Clustering. In T. Sarjakoski, M. Y. Santos, \& L. T. Sarjakoski (Eds.), Geospatial Data in a Changing World (pp. 199-218). doi: 10.1007/978-3-319-33783$8 \_12$

Bonnel, P., Bayart, C., \& Smith, B. (2015). ScienceDirect Workshop Synthesis: Comparing and combining survey modes. Transportation Research Procedia, 11, 108-117. doi: 10.1016/j.trpro.2015.12.010

Boss, D., Nelson, T., Winters, M., \& Ferster, C. J. (2018). Using crowdsourced data to monitor change in spatial patterns of bicycle ridership. Journal of Transport \& Health. doi: 10.1016/j.jth.2018.02.008

Brennan, T. M., Gurriell, R. A., Bechtel, A. J., \& Venigalla, M. M. (2019). Visualizing and Evaluating Interdependent Regional Traffic Congestion and System Resiliency, a Case Study Using Big Data from Probe Vehicles. Journal of Big Data Analytics in Transportation, 1(1), 25-36. doi: 10.1007/s42421-019-00002-y

Buehler, R. (2018). Can Public Transportation Compete with Automated and Connected Cars? Journal of Public Transportation, 21(1), 7-18. doi: 10.5038/2375-0901.21.1.2

Chen, C., Ma, J., Susilo, Y., Liu, Y., \& Wang, M. (2016). The promises of big data and small 
data for travel behavior (aka human mobility) analysis. Transportation Research Part C: Emerging Technologies, 68, 285299. doi: $10.1016 /$ j.trc.2016.04.005

Chen, X., Zahiri, M., \& Zhang, S. (2017). Understanding ridesplitting behavior of ondemand ride services: An ensemble learning approach. Transportation Research Part $C$, 76, 51-70. doi: 10.1016/j.trc.2016.12.018

Crawford, K. (2013). The Hidden Biases in Big Data. Retrieved August 4, 2017, from Harvard Business Review website: https://hbr.org/2013/04/the-hidden-biasesin-big-data

Crayton, T. J., \& Meier, B. M. (2017). Autonomous vehicles: Developing a public health research agenda to frame the future of transportation policy. Journal of Transport \& Health, 6(April), 245-252. doi: 10.1016/j.jth.2017.04.004

Desouza, K. C., \& Smith, K. L. (2016). PAS Report 585 Big Data and Planning. In $P A S$ Report.

doi: http://dx.doi.org/10.1007/s13222-0160214-9

Diao, M., Zhu, Y., Ferreira, J., \& Ratti, C. (2016). Inferring individual daily activities from mobile phone traces: A Boston example. Environment and Planning B: Planning and Design, 43(5), 920-940. doi: $10.1177 / 0265813515600896$

Efthymiou, A., Barmpounakis, E. N., Efthymiou, D., \& Vlahogianni, E. I. (2019). Transportation Mode Detection from LowPower Smartphone Sensors Using TreeBased Ensembles. Journal of Big Data Analytics in Transportation, 1(1), 57-69. doi: 10.1007/s42421-019-00004-w

Erhardt, G. D., \& Dennett, A. (2017). Understanding the Role and Relevance of the Census in a Changing Transportation Data Landscape. Transportation Research Board Conference on Applying Census Data for Transportation. Retrieved from http://onlinepubs.trb.org/onlinepubs/confer
ences/2017/censusdata/KeepingCensusRel evant.pdf

Evans-Cowley, J. S., \& Griffin, G. P. (2012). Microparticipation with Social Media for Community Engagement in Transportation Planning. Transportation Research Record: Journal of the Transportation Research Board, 2307(1), 90-98. doi: 10.3141/230710

Fagnant, D. J., \& Kockelman, K. M. (2014). The travel and environmental implications of shared autonomous vehicles, using agentbased model scenarios. Transportation Research Part C: Emerging Technologies, 40(2014), 1-13. doi: $10.1016 / j \cdot \operatorname{trc} .2013 .12 .001$

García-Albertos, P., Picornell, M., Salas-Olmedo, M. H., \& Gutiérrez, J. (2018). Exploring the potential of mobile phone records and online route planners for dynamic accessibility analysis. Transportation Research Part A: Policy and Practice. doi: 10.1016/j.tra.2018.02.008

Garmin. (2018). Garmin Connect. Retrieved May 31, 2018, from https://connect.garmin.com/en-US/

Griffin, G. P., \& Jiao, J. (2015a). Crowdsourcing Bicycle Volumes: Exploring the role of volunteered geographic information and established monitoring methods. URISA Journal, 27(1), 57-66. Retrieved from $\mathrm{http}: / /$ soa.utexas.edu/sites/default/disk/Grif fin-Crowdsourcing_Bicycle_Volumes.pdf

Griffin, G. P., \& Jiao, J. (2015b). Where does bicycling for health happen? Analysing volunteered geographic information through place and plexus. Journal of Transport \& Health, 2(2), 238-247. doi: 10.1016/j.jth.2014.12.001

Griffin, G. P., Nordback, K., Götschi, T., Stolz, E., \& Kothuri, S. (2014). Monitoring Bicyclist and Pedestrian Travel and Behavior, Transportation Research Circular E-C183. doi: $10.17226 / 22420$ 
Gschwender, A., Munizaga, M., \& Simonetti, C. (2016). Using smart card and GPS data for policy and planning: The case of Transantiago. Research in Transportation Economics, 59, 242-249. doi: 10.1016/j.retrec.2016.05.004

Guerra, E., \& Morris, E. A. (2018). Cities, Automation, and the Self-parking Elephant in the Room. Planning Theory and Practice, 9357, 1-7. doi: $10.1080 / 14649357.2017 .1416776$

Guest, G., MacQueen, K., \& Namey, E. (2014). Applied Thematic Analysis. doi: 10.4135/9781483384436

Gürbüz, F., \& Turna, F. (2018). Rule extraction for tram faults via data mining for safe transportation. Transportation Research Part A: Policy and Practice, 116(June), 568-579. doi: 10.1016/j.tra.2018.07.011

Harris, R., O'Sullivan, D., Gahegan, M., Charlton, M., Comber, L., Longley, P., ... Evans, A. (2017). More bark than bytes? Reflections on 21+ years of geocomputation. Environment and Planning B: Urban Analytics and City Science, 44(4), 598-617. doi: $10.1177 / 2399808317710132$

Henke, N., Bughin, J., Chui, M., Manyika, J., Saleh, T., Wiseman, B., \& Sethupathy, G. (2016). The Age of Analytics : Competing in a Data-Driven World. In McKinsey Global Institute (Vol. 12). doi: 10.1111/bjet.12230

Hipp, J. A., Adlakha, D., Eyler, A. A., Gernes, R., Kargol, A., Stylianou, A. H., \& Pless, R. (2017). Learning from Outdoor Webcams: Surveillance of Physical Activity Across Environments. In P. (Vonu) Hakuriah, N. Tilahun, \& M. Zellner (Eds.), Seeing Cities Through Big Data (pp. 471-490). doi: 10.1007/978-3-319-40902-3_26

Hong, A., Kim, B., \& Widener, M. (2019). Noise and the city: Leveraging crowdsourced big data to examine the spatio-temporal relationship between urban development and noise annoyance. Environment and Planning B: Urban Analytics and City Science. doi: 10.1177/2399808318821112

Johnson, T. P., \& Smith, T. W. (2017). Big Data and Survey Research: Supplement or Substitute? In P. (Vonu) Hakuriah, N. Tilahun, \& M. Zellner (Eds.), Seeing Cities Through Big Data (pp. 113-125). doi: 10.1007/978-3-319-40902-3 7

Kieu, L. M., Bhaskar, A., \& Chung, E. (2015). Passenger segmentation using smart card data. IEEE Transactions on Intelligent Transportation Systems, 16(3), 1537-1548. doi: 10.1109/TITS.2014.2368998

Kitchel, D., \& Riordan, B. (2014). Strava Metro Product Documentation. Hanover, NH.

Krippendorff, K., \& Craggs, R. (2016). The Reliability of Multi-Valued Coding of Data. Communication Methods and Measures, 10(4), 181-198. doi: $10.1080 / 19312458.2016 .1228863$

Krishnamurthy, R., Smith, K. L., \& Desouza, K. C. (2017). Urban Informatics: Critical Data and Technology Considerations. In P. (Vonu) Hakuriah, N. Tilahun, \& M. Zellner (Eds.), Seeing Cities Through Big Data (pp. 163-188). doi: 10.1007/978-3-319-40902$3 \_10$

Kwan, M.-P. (2012). The Uncertain Geographic Context Problem. Annals of the Association of American Geographers, 102(5), 958968. doi: 10.1080/00045608.2012.687349

Legacy, C., Ashmore, D., Scheurer, J., Stone, J., $\&$ Curtis, C. (2019). Planning the driverless city. Transport Reviews, 39(1), 84-102. doi: 10.1080/01441647.2018.1466835

Mcardle, G., \& Kitchin, R. (2016). Improving the Veracity of Open and Real-Time Urban Data. Built Environment, 42(3), 457-473. doi: 10.2148/benv.42.3.457

Mehmood, R., Meriton, R., Graham, G., Hennelly, P., \& Kumar, M. (2017). Exploring the influence of big data on city 
transport operations: a Markovian approach. International Journal of Operations \& Production Management, 37(1), 75-104. doi: 10.1108/IJOPM-032015-0179

Mondschein, A. (2015). Five-star transportation: using online activity reviews to examine mode choice to non-work destinations. Transportation, 42(4), 707-722. doi: 10.1007/s11116-015-9600-7

Murphy, J., Link, M. W., Childs, J. H., Tesfaye, C. L., Dean, E., Stern, M., ... Harwood, P. (2014). Social Media in Public Opinion Research : Report of the AAPOR Task Force on Emerging Technologies in Public Opinion Research. Deerfield, IL.

O'Connor, H., Madge, C., Shaw, R., \& Wellens, J. (2008). Internet-based Interviewing. In N. Fielding, R. M. Lee, \& G. Blank (Eds.), The SAGE Handbook of Online Research Methods (pp. 271-289). doi: 10.4135/9780857020055

Ose, S. O. (2016). Using Excel and Word to Structure Qualitative Data. Journal of Applied Social Science, 10(2), 147-162. doi: $10.1177 / 1936724416664948$

Peng, P., Yang, Y., Lu, F., Cheng, S., Mou, N., \& Yang, R. (2018). Modelling the competitiveness of the ports along the Maritime Silk Road with big data. Transportation Research Part A: Policy and Practice, 118(October), 852-867. doi: 10.1016/j.tra.2018.10.041

Piwek, L., Joinson, A., \& Morvan, J. (2015). The use of self-monitoring solutions amongst cyclists: An online survey and empirical study. Transportation Research Part A: Policy and Practice, 77, 126-136. doi: 10.1016/j.tra.2015.04.010

Schweitzer, L. A. (2014). Planning and Social Media: A Case Study of Public Transit and Stigma on Twitter. Journal of the American Planning Association, 80(3), 218-238. doi: 10.1080/01944363.2014.980439
Schweitzer, L. A., \& Afzalan, N. (2017). Four Reasons Why AICP Needs an Open Data Ethic. Journal of the American Planning Association, 83(2), 161-167. doi: 10.1080/01944363.2017.1290495

Sener, I. N., Zmud, J., \& Simek, C. (2018). Examining Future Automated Vehicle Usage A Focus on the Role of Ride Hailing. Retrieved from https://static.tti.tamu.edu/tti.tamu.edu/docu ments/TTI-2018-2.pdf

Shearmur, R. (2015). Dazzled by data: Big Data, the census and urban geography. Urban Geography, (August 2015), 1-4. doi: 10.1080/02723638.2015.1050922

Smith, W. R. (2017). Communication, Sportsmanship, and Negotiating Ethical Conduct on the Digital Playing Field. Communication \& Sport, 5(2), 160-185. doi: $10.1177 / 2167479515600199$

Stenneth, L., Wolfson, O., Yu, P. S., \& Xu, B. (2011). Transportation mode detection using mobile phones and GIS information. Proceedings of the 19th ACM SIGSPATIAL International Conference on Advances in Geographic Information Systems - GIS '11, 54. doi: $10.1145 / 2093973.2093982$

Tasse, D., \& Hong, J. I. (2017). Using UserGenerated Content to Understand Cities. In P. (Vonu) Hakuriah, N. Tilahun, \& M. Zellner (Eds.), Seeing Cities Through Big Data (pp. 49-64). doi: 10.1007/978-3-31940902-3_3

Taylor, L. (2016). No place to hide? The ethics and analytics of tracking mobility using mobile phone data. Environment and Planning D: Society and Space, 34(2), 319336. doi: $10.1177 / 0263775815608851$

Teddlie, C., \& Tashakkori, A. (2010). Overview of Contemporary Issues in Mixed Methods Research. In SAGE Handbook of Mixed Methods in Social \& Behavioral Research (pp. 1-42). doi: 10.4135/9781506335193.n1 
Toole, J. L., Colak, S., Sturt, B., Alexander, L. P., Evsukoff, A., \& González, M. C. (2015). The path most traveled: Travel demand estimation using big data resources. Transportation Research Part C: Emerging Technologies, 58, 162-177. doi: 10.1016/j.trc.2015.04.022

Vij, A., \& Shankari, K. (2015). When is big data big enough? Implications of using GPSbased surveys for travel demand analysis. Transportation Research Part C, 56, 446462. doi: 10.1016/j.trc.2015.04.025

Wagh, A., Li, X., Sudhaakar, R., Addepalli, S., \& Qiao, C. (2013). Data fusion with flexible message composition in Driver-in-the-Loop vehicular CPS. Ad Hoc Networks, 11(7), 2083-2095. doi: 10.1016/j.adhoc.2012.02.012

Xie, K., Yang, D., Ozbay, K., \& Yang, H. (2019). Use of real-world connected vehicle data in identifying high-risk locations based on a new surrogate safety measure. Accident Analysis and Prevention, 125(July 2018), 311-319. doi: 10.1016/j.aap.2018.07.002

Yin, L., Cheng, Q., Shao, Z., Wang, Z., \& Wu, L. (2017). 'Big Data': Pedestrian Volume Using Google Street View Images. In P. (Vonu) Hakuriah, N. Tilahun, \& M. Zellner (Eds.), Seeing Cities Through Big Data (pp. 461-469). doi: 10.1007/978-3-319-409023_25

Zhang, Z., \& He, Q. (2019). Social Media in Transportation Research and Promising Applications. In S. V. Ukkusuri \& Y. Chao (Eds.), Transportation Analytics in the Era ofBig Data (pp. 23-45). doi: 10.1007/9783-319-75862-6_2

Zhao, J., Wang, J., Xing, Z., Luan, X., \& Jiang, Y. (2018). Weather and cycling: Mining big data to have an in-depth understanding of the association of weather variability with cycling on an off-road trail and an on-road bike lane. Transportation Research Part A: Policy and Practice, 111(March), 119-135. doi: 10.1016/j.tra.2018.03.001

Zhao, M., Venkatanarayana, R., \& Fontaine, M. D. (2017). Development of a Framework for VDOT Big Data Analytics Technical Assistance Final Report.

Zhou, X., Chen, Z., Yeh, A. G. O., \& Yue, Y. (2019). Workplace segregation of rural migrants in urban China: A case study of Shenzhen using cellphone big data. Environment and Planning B: Urban Analytics and City Science. doi: 10.1177/2399808319846903

Zhou, X., Yeh, A. G. O., Li, W., \& Yue, Y. (2018). A commuting spectrum analysis of the jobshousing balance and self-containment of employment with mobile phone location big data. Environment and Planning B: Urban Analytics and City Science, 45(3), 434-451. doi: $10.1177 / 2399808317707967$

Zmud, J., Williams, T., Outwater, M., Bradley, M., Kalra, N., \& Row, S. (2018). Updating Regional Transportation Planning and Modeling Tools to Address Impacts of Connected and Automated Vehicles, Volume 2: Guidance. In Updating Regional Transportation Planning and Modeling Tools to Address Impacts of Connected and Automated Vehicles, Volume 2: Guidance. doi: $10.17226 / 25332$

\section{Acknowledgements}

The authors appreciate interview coding support by Boya Dai with the Texas A\&M Transportation Institute, and insightful comments from four reviewers.

This project was supported by the Safety through Disruption (Safe-D) National University Transportation Center, a grant from the U.S. Department of Transportation - Office of the Assistant Secretary for Research and Technology, University Transportation Centers Program. 